\title{
USO DO ÁCIDO TRANEXÂMICO EM RESSUSCITAÇÃO TRAUMÁTICA NO AMBIENTE PRÉ-HOSPITALAR: RELATO DE CASO
}

\author{
Lilyan $^{1}$ Paula de Sousa Teixeira LIMA ${ }^{1}$; Herberth Jessie MARTINS²; Daniel Augusto \\ de Souza RODRIGUES ${ }^{3}$; Paulo Regis Souza SANTOS ${ }^{4}$
}

\section{RESUMO}

Este relato descreve o uso do ácido tranexâmico em situação de choque hemorrágico na abordagem inicial ao paciente vítima de trauma. O caso descreve a atuação da equipe de resgate aéreo em apoio à unidade de atendimento préhospitalar fixa no atendimento a homem jovem com múltiplas perfurações em tórax por arma branca com evolução rápida à condição de choque hipovolêmico grave e posterior transporte à unidade especializada com centro de trauma. Justificativa: o serviço aéreo de resgate é uma importante ferramenta quando o assunto é agilidade para atendimentos aos casos de vítimas em grave estado de saúde, como nas situações de choque hipovolêmico causados por hemorragia. Para essa condição clínica é fundamental que se inicie a administração endovenosa precoce de ácido tranexâmico. Objetivos: descrever o uso do ácido tranexâmico em paciente vítima de trauma com hemorragia severa e a importância do serviço de suporte avançado de vida no rápido transporte de paciente para unidade de saúde especializada. Método: trata-se de um relato de experiência baseado em atendimento inicial a paciente vítima de trauma por múltiplas perfurações por arma branca em tórax realizado por serviço aéreo de resgate em conjunto com Unidade de Pronto Atendimento - UPA com posterior transporte para tratamento definitivo em centro de trauma de referência. Resultados: a vítima foi transportada após ser estabilizada hemodinamicamente pela equipe do serviço de resgate aéreo. Conclusão: $A$ administração endovenosa precoce de ácido tranexâmico repercute positivamente no prognóstico de vítimas de trauma com hemorragia severa.

Palavras-chave: resgate aéreo, choque hemorrágico, ácido tranexâmico.

\section{INTRODUÇÃO}

O trauma é apontado como a principal causa de morte na população brasileira entre 1 e 49 anos de vida (BRASIL, 2021). Quando associado ao processo de coagulopatia, incluindo hiperfibrinólise, o trauma eleva a chance de desfecho desfavorável, sendo os processos hemorrágicos a causa de muitos óbitos por traumatismo (SCHOBER et al., 2019; NEEKI et al., 2017; LUZ et al., 2012).

\footnotetext{
11 Enfermeira, Mestre em Cuidado, Gestão e Tecnologia em Saúde e Enfermagem. SAMU-DF, lilyanpls@gmail.com

2 Enfermeiro, Pós-graduação em Enfermagem Aeroespacial, SAMU-DF, herberthmartins2012@gmail.com

3 Médico, Cirurgião vascular, CBMDF, dangutus@yahoo.com.br

4 Médico, Clínica Médica, SAMU-DF, paulo_regisba@hotmail.com
} 
O ácido tranexâmico (TXA) é uma medicação capaz de reduzir o sangramento ao inibir competitivamente a fibrinólise e a formação da fibrina através inativação do plasminogênio conforme descrito por Shakur et al., (2010), Spahn et al., (2019) e Thiengo et al., V. (2020).

Após a publicação do estudo CRASH2 em 2010, o manejo inicial do choque hemorrágico grave com administração de $1 \mathrm{~g}$ intravenoso em até 3 horas de TXA foi difundido mundialmente. Sua importância é também descrita em hemorragia pósparto segundo Shakur et al., (2017) demonstrado no estudo WOMAN e em cirurgia cardíaca conforme Myles et al., (2017) demonstrado no estudo ATACAS.

Pesquisas mais recentes, ao relacionar o óbito à hiperfibrinólise, recomendam o uso de TXA, preferencialmente por via intravenosa, o mais precoce possível em vítimas de trauma, favorecendo seu uso no ambiente pré-hospitalar, conforme vemos nos estudos CRASH-2 (2011) e CRASH-3 (2019).

O presente artigo relata a experiência de uma equipe de resgate aéreo em cenário pré-hospitalar no uso do TXA em processo hemorrágico grave relacionado ao trauma. Neste relato demonstramos as respostas rápidas e objetivas frente à condição clínica, assim como o planejamento e comunicação entre os serviços de saúde pré-hospitalares e intra-hospitalares.

\section{METODOLOGIA}

Esse relato de experiência descreve um atendimento a paciente do sexo masculino com 19 anos, vítima de trauma por múltiplas perfurações em tórax por arma branca no mês de dezembro de 2020. Os familiares levaram o paciente para a Unidade de Pronto Atendimento (UPA) e este apresentava-se consciente, orientado, taquidispnéico, cianótico, com sangramento ativo em três perfurações em tórax anterior (1 perfuração em hemitórax superior direito e duas perfurações em hemitórax esquerdo).

$\mathrm{Na}$ UPA houve hidratação vigorosa com Solução Fisiológica $0,9 \%$ e Ringer Lactato totalizando 5 litros, oxigenioterapia com máscara não reinalante e curativo valvular em perfurações em tórax.

O Centro de Trauma de referência da região foi informado, e o serviço aéreo foi acionado para transporte de unidade de baixa complexidade para alta complexidade. 
$\mathrm{Na}$ chegada da equipe do resgate aéreo com 35 minutos após o trauma, foi identificada condição de choque grau IV não compensado por reposição volêmica e sangramento ativo em perfurações torácicas. Optou-se pela administração imediata 1g de TXA e inserção de várias gazes na lesão em região infraescapular esquerda, bloqueando o sangramento que posteriormente foi identificada em tomografia como lesão puntiforme da artéria subclávia. Havia pneumotórax aberto que após intubação orotraqueal, evoluiu para pneumotórax hipertensivo sendo necessário toracostomia. O paciente foi estabilizado hemodinamicamente e preparado para transporte.

Durante o transporte, após 15 minutos da infusão do TXA, pode ser observada redução significativa do sangramento. Após 40 minutos da infusão não foi observado mais sangramento, correspondendo com melhora de sinais clínicos com presença de pulso periférico, elevação da pressão arterial de $70 \times 40 \mathrm{mmHg}$ para $105 \times 70 \mathrm{mmHg}$.

\section{RESULTADOS E DISCUSSÕES}

Ensaios clínicos randomizados, coortes e metanálises têm sido realizados a fim de avaliar o custo efetividade do uso do TXA no atendimento inicial ao trauma na condição de choque hemorrágico (COATS et al., 2011; CRASH-2, 2011; ROBERTS et al., 2012; SOBRAL, 2016), entretanto pouco tem sido estudado sobre o manejo dessa medicação na realidade brasileira (GUERRIERO, 2011).

A estratégia inicial nessa descrtição coaduna com a literatura na prerrogativa de controle inicial ao choque hemorrágico com contenção do sangramento e reposição de fluidos, limitado aos recursos disponíveis no local e pela indisponibilidade de hemotransfusão (AMERICAN COLLEGE OF SURGEONS, 2018).

É reconhecido o efeito deletério de administração excessiva de cristalóides e a importância de abordagem precoce para tratamento da coagulopatia induzida por trauma (CALLUM et al., 2016). Nessa perspectiva, o ácido tranexâmico apresentouse como uma alternativa promissora (CARREIRO, 2014).

O uso do TXA nessa experiência compartilhada se assemelha à indicação encontrada em estudo similar, cuja intervenção foi bem sucedida em condição de hipotensão permissiva, ferimento em tórax, infusão do TXA em tempo médio de 32 minutos e transporte aéreo como opção de evacuação mais rápida (VU, 2013). 
Sua indicação para redução de mortalidade é mais evidenciada em situações de trauma severo e com coagulopatia já instalada (CRASH-2, 2011; HARVEY, PERRONE, KIM, 2014; MEJIA-MANTILLA et al., 2009).

A eficácia do TXA está bem estabelecida no trauma - nível de evidência $A$ (CRASH-2, 2011; ROBERTS et al., 2012). Já no cenário pré-hospitalar ainda não apresenta níveis de evidência elevados - nível de evidência C (LIPSKY et al., 2014; VU, 2013), necessitando de estudos que melhor discriminem os aspectos epidemiológicos dessa prática clínica.

\section{CONSIDERAÇÕES FINAIS}

A experiência apresenta uma situação sugestiva de eficácia bem estabelecida ao uso do TXA em vítima de trauma com coagulopatia induzida por choque hemorrágico grave. As medidas adotadas pela equipe de resgate aéreo, tanto no manejo do TXA, como nas medidas de contenção do sangramento foram fundamentais para a ressuscitação e estabilização hemodinâmica do paciente.

Ainda não se pode afirmar que o TXA tem real benefício na coagulopatia póstraumática quando utilizado em ambiente pré-hospitalar. Estudos evidenciam a efetividade do TXA associada à precocidade no seu uso, porém há necessidade de mais estudos que alcancem o paciente na perspectiva de atendimento pré-hospitalar a fim de avaliar a segurança do tratamento nesse cenário. 


\section{REFERÊNCIAS BIBLIOGRÁFICAS}

\section{AMERICAN COLLEGE OF SURGEONS. ATLS - Advanced Trauma Life Support} for Doctors. 10. ed. Chicago: Committee on Trauma, 2018. 474 p.

BRASIL. Ministério da Saúde. Informação de Ministério da Saúde. Disponível em: http://tabnet.datasus.gov.br/cgi/tabcgi.exe?sim/cnv/ext10uf.def. Acesso em 3 mar. 2021.

CALLUM, J. L. et al. Bloody easy 4: blood transfusion, blood alternatives and transfusion reactions: a guide to transfusion medicine. Toronto: Ontário Regional Blood Coordinating Network, 2016. Disponível em: https://transfusionontario.org/en/bloody-easy-4-blood-transfusions-blood-alternativesand-transfusion-reactions-a-guide-to-transfusion-medicine-fourth-edition/ Acesso em: 3 fev. 2021.

CARREIRO, P. R. L. Hipotensão Permissiva no Trauma. Revista Médica de Minas Gerais, Belo Horizonte, v. 24, n. 4, p 515-519, 2014. Disponível em: https://pesquisa.bvsalud.org/portal/resource/pt/lil-749276. Acesso em: 5 fev. 2021.

COATS, T. et al. Antifibrinolytic drugs for acute traumatic injury (Review). The Cochrane Collaboration. Medical Journal, São Paulo, v. 129, n. 5, 2011. Disponível em: https://www.scielo.br/j/spmj/a/7LRBwkDNdp4D8qpdvcnWv9H/?lang=en Acesso em: 4 mar. 2021.

GUERRIERO, C. et al. Cost-effectiveness analysis of administering tranexamic acid to bleeding trauma patients using evidence from the CRASH-2 trial. PLoS One, [s.L.], v. $\quad 6, \quad$ n. $5, \quad 3$ maio. 2011. Disponível em: https://journals.plos.org/plosone/article?id=10.1371/journal.pone.0018987 Acesso em: 20 abr. 2021.

HARVEY, V.; PERRONE, J.; KIM, P. Does the use of tranexamic acid improve trauma mortality? Ann Emerg Med, [s.L.], v. 63, n. 4, p.460-462, 2014. Disponível em: 
https://www.annemergmed.com/article/S0196-0644(13)01341-3/fulltext. Acesso em: 5 jan. 2021

LIPSKY, A.M. et al. Tranexamic acid in the prehospital setting: Israel Defense Forces' initial experience. Injury, [s.L.], v. 45, n.1, p.66-70, 2014. Disponível em: https://pubmed.ncbi.nlm.nih.gov/24074828/. Acesso em: 10 fev. 2021.

LUZ L, et al. Ácido tranexâmico no tratamento da hemorragia no trauma. Revista do Colégio Brasileiro de Cirurgiões, [s.L.], v. 39, n. 1, p. 077-080, 2012. Disponível em: https://www.scielo.br/j/rcbc/a/p6fjjp6ccTX9JdJp4PXcgVG/?lang=pt. Acesso em: 10 abr 2021.

MEJIA-MANTILLA, JH et al. Hemorrhage and trauma, advances in the CRASH-2 study in Colombia. Revista Colombiana de Cirurgia, [s.L.], v. 24, n. 3, p. 175-183. 2009. Disponível em: http://www.scielo.org.co/scielo.php?script=sci_abt\&pid=S201175822009000300006. Acesso em: 2 abr. 2021.

MYLES, P.S. et al. Tranexamic acid in patients undergoing coronary-artery surgery. The New England Journal of Medicine, [s.L.], v. 376, n. 2, p. 136-148, 2017. Disponível em: https://www.nejm.org/doi/pdf/10.1056/NEJMoa1606424. Acesso em 28 fev. 2021

NEEKI, M.M. et al. Efficacy and safety of tranexamic acid in prehospital traumatic hemorrhagic shock: outcomes of the Cal-PAT study. Western Journal Emergency Medicine, [s.L.], v. 18, p. 673-683. 19 abr. 2017. Disponível em: https://pubmed.ncbi.nlm.nih.gov/28611888/. Acesso em: 3 abr. 2021.

ROBERTS, I. et. al. Antifibrinolytic drugs for acute traumatic injury. The Cochrane Database Systematic Reviews, [s.L.], n. 12. 2012. Disponível em: https://pubmed.ncbi.nlm.nih.gov/23418644/. Acesso em: 10 abr. 2021.

SCHOBER, P. et al. Hemorrhage treatment adjuncts in a helicopter Emergency Medical Service. Air Medical Journal, v. 38, n. 3, p. 209-211, 2019. Disponível em: 
https://www.airmedicaljournal.com/article/S1067-991X(18)30156-1/fulltext. Acesso em: 3 fev. 2021.

SHAKUR, H. et al. Effects of tranexamic acid on death, vascular occlusive events, and blood transfusion in trauma patients with significant haemorrhage. Lancet. [s.L.], v. $376, \quad$ n. 9734 , p. 23-32, jun. 2010. Disponível em: https://www.thelancet.com/journals/lancet/article/PIIS0140-6736(10)60835-5/fulltext. Acesso em: 20 mar. 2021.

SHAKUR, H. et al. Effect of early tranexamic acid administration on mortality, hysterectomy, and other morbidities in women with post-partum haemorrhage (WOMAN). Lancet, [s.L.], v. 389, n. 10084, p. 2105-16, abr. 2017. Disponível em: https://pubmed.ncbi.nlm.nih.gov/28456509/. Acesso em: 20 fev. 2021.

SOBRAL, F. A. et al. Tranexamic acid action on liver regeneration after partial hepatectomy: experimental model in rats. Arquivos Brasileiros de Cirurgia Digestiva, [s.L.], $\quad$ v. $29, \quad$ n. $\quad 2, \quad$ p.102-104, 2016. Disponível em: https://www.scielo.br/j/abcd/a/j9bk6crcmQD5VMMNJGvZt7y/?lang=pt\&format=html. Acesso em: 27 jan. 2021.

SPAHN, D. R. The European guideline on management of major bleeding and coagulopathy following trauma. Critical Care, [s.L.], v. 23, n. 98, 2019. Disponível em: https://ccforum.biomedcentral.com/articles/10.1186/s13054-019-2347-3. Acesso em: 20 fev. 2021.

The CRASH-2 collaborators. The importance of early treatment with tranexamic acid in bleeding trauma patients: an exploratory analysis of the CRASH-2 randomised controlled trial. Lancet. [s.L.], v. 377, n. 9771 p. 1096-101, 2011. Disponível em: https://pubmed.ncbi.nlm.nih.gov/21439633/. Acesso em: 10 mar. 2021.

The CRASH-3 trials collaborators. Effects of tranexamic acid on death, disability, vascular occlusive events and other morbidities in patients with acute traumatic brain injury (CRASH-3): a randomised, placebo-controlled trial. Lancet. [s.L.], v. 394, n. 10210, p. $1713 \quad-\quad 23$, out. 2019. Disponível em: 
evoluigi

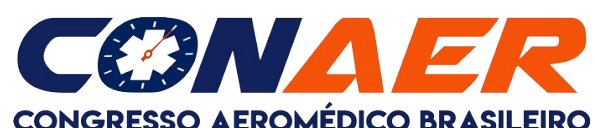

https://www.thelancet.com/journals/lancet/article/PIIS0140-6736(19)32233-0/fulltext Acesso em: 2 mar. 2021.

THIENGO, V. F. et al. Uso pré-hospitalar do ácido tranexâmico: uma revisão da literatura. Revista de Medicina, São Paulo. v. 99, n. 1, p.62-5, 2020. Disponível em: https://www.revistas.usp.br/revistadc/article/view/164150. Acesso em: 20 fev. 2021.

VU, E. N. Prehospital use of tranexamic acid for hemorrhagic shock in primary and secondary air medical evacuation. Air Medical Journal, [s.L.], v. 32, n. 5, p. 289-92, 2013. Disponível em: https://pubmed.ncbi.nlm.nih.gov/24001917/. Acesso em: 20 mar. 2021. 